\title{
Neuroimage
}

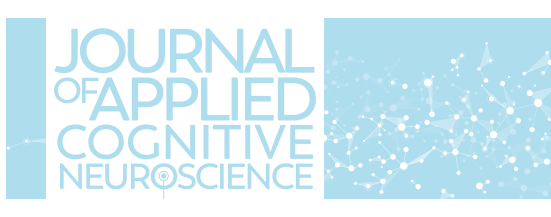

\section{A look back into a typical patient with memory complains}

DOI: https://doi.org/10.17981/JACN.1.1.2020.09

Patricio Alexis Chrem-Méndez ${ }^{1} \&$ Silvia Vázquez ${ }^{1}$

${ }^{1}$ Neurology department, Fleni (Buenos Aires, Argentina)

Correspondence:

\section{Patricio Alexis}

\section{Chrem-Mendez}

Neurology department, Fleni (Buenos Aires, Argentina)

E mail:

pchremmendez@fleni.org.ar
Aim: The purpose of these images is to extol the biological perspective from the clinical setting perspective of a typical AD case. This participant was profoundly studied and also followed-up for five years in the study ADNI- Argentina.

Clinical setting: The participant was 72 years old woman with an amnestic Mild Cognitive Impairment (MCI). She was enrolled in 2013, following the ADNI2 criteria as Late MCI. Her APOE status was $3 / 4$, and scored 27/30 in the MMSE and 0.5 in CDR at baseline.

Biological Findings in CSF at Baseline: AB42 $447 \mathrm{pg} / \mathrm{ml}$; T-tau $599 \mathrm{pg} / \mathrm{ml} ;$ P-tau $103 \mathrm{pg} / \mathrm{ml}$; Nfl $1124 \mathrm{pg} / \mathrm{ml}$.

MRI at baseline: MTA 3/2, Fazekas 0, Koedam 1, Hippocampus volumes: L 2691, R 2318.

Conclusion: The molecular imaging revealed, beyond the clinician's eye, all the phenomena that had preceded the first clinical symptom. According to the new ATN scheme, the participant was positive in every aspect, and most probably, the whole process could have lasted around 15 years before its enrollment. After 60 months of follow-up the participant's MMSE was 15 and de CDR was 1 . Sometimes an image can say a thousand words about the time that had elapsed when the physician first met the patient. 


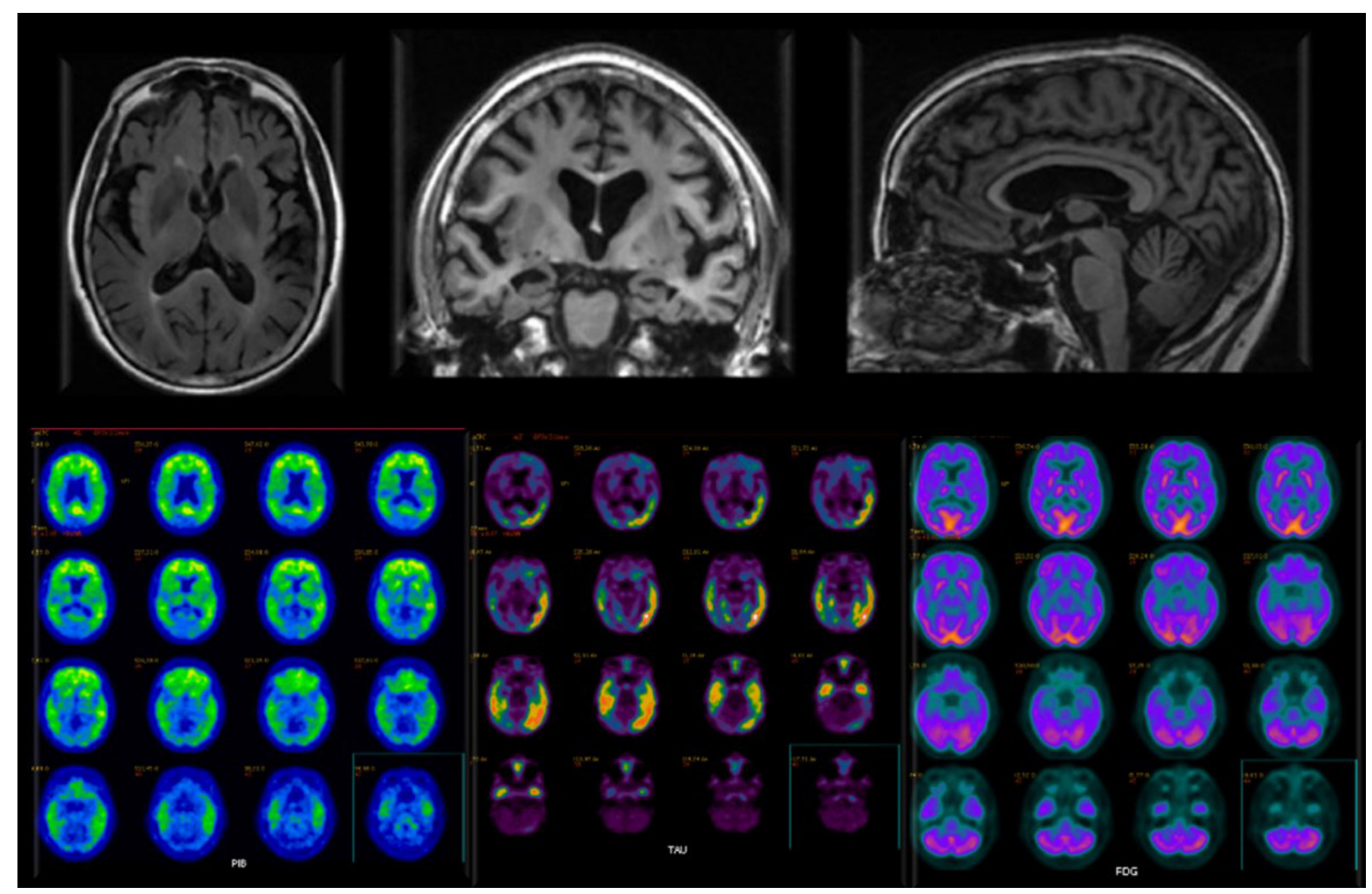

Figura 1. PET-PiB: A+, Tau-PET: T+, PET-FDG: N+ Source: Authors.

\section{BIBLIOGRAPHY}

Aisen, P. S., Petersen, R. C., Donohue, M. \& Weiner, M. W. (2015). Alzheimer's Disease Neuroimaging Initiative. Alzheimer's Disease Neuroimaging Initiative 2 Clinical Core: Progress and plans. Alzheimers Dement, 11(7), 734-739. https://doi.org/10.1016/j. jalz.2015.05.005

Clifford, J., Bennett, D., Blennow, K., Carrillo, M., Dunn, B., Budd, S., Holtzman, D., Jagust, W., Jessen, F., Karlawish, J., Liu, E., Molinuevo, J., Montine, T., Phelps, C., Rankin, K., Rowe, C., Scheltens, P., Siemers, E., Snyder, H. \& Sperling, R. (2018). NIA-AA Research Framework: Toward a biological definition of Alzheimer's disease. Alzheimer's \& Dementia, 14(4), 535-562. https:// doi.org/10.1016/j.jalz.2018.02.018
Méndez, P., Calandri, I., Nahas, F., Russo, M., Demey, I., Martín, M., Clarens, M., Harris, P., Tapajoz, F., Campos, J., Surace, E., Martinetto, H., Ventrice, F., Cohen, G., Vázquez, S., Romero, C., Guinjoan, S., Allegri, R. \& Sevlever, G. (2018). Argentina-Alzheimer's disease neuroimaging initiative (Arg-ADNI): neuropsychological evolution profile after one-year follow up. Arquivos de Neuropsiquiatria, 76(4), 231-240. https:// doi.org/10.1590/0004-282x20180025

Russo, M., Gustafson, D., Vázquez, S., Surace, E., Guinjoan, S., Allegri, R. F. \& Sevlever, G. (2014). ArgentinaAlzheimer's Disease Neuroimaging Initiative. Creation of the ArgentinaAlzheimer's Disease Neuroimaging Initiative. Alzheimer's \& Dementia, 10(1S), 84-87. https://doi.org/10.1016/j. jalz.2013.09.015 
Patrico A. Chrem Méndez: Neurologist.

Siliva Vásquez: Medical Director Center for Molecular Imaging CIM (Buenos Aires, Argentina). 